\title{
Urban, semi-urban and rural difference in the prevalence of metabolic syndrome in Shaanxi province, northwestern China: a population-based survey
}

Shaoyong Xu', Jie Ming ${ }^{1}$, Chao Yang ${ }^{2}$, Bin Gao ${ }^{1}$, Yi Wan ${ }^{3}$, Ying Xing ${ }^{1}$, Lei Zhang ${ }^{4}$ and Qiuhe $\mathrm{Ji}^{i^{*}}$

\begin{abstract}
Background: The ongoing rapid urbanization in China offers rural population opportunities not only for economic improvement but also for substantial health risks. Albeit some researches related to rural-urban difference of metabolic syndrome (MS), there lacks studies focusing on this point in undeveloped provinces in China.

Methods: The survey, as part of China National Diabetes and Metabolic disorders Study, was conducted in Shaanxi province from June 2007 to May 2008. A total of 3,297 adults aged 20 years or older were included, of which 1,467 individuals were from urban areas, 839 from semi-urban areas, and 890 from rural areas. The MS was defined according to the 2009 Joint Interim Statement.

Results: The age-standardized prevalence of MS was significant higher in rural residents than in urban counterparts ( $29.0 \%$ vs. $25.9 \%, P=0.017)$, in particular among females (30.2\% vs. $24.4 \%, P=0.003)$. After adjusted for the listed risk factors, rural residents had a $27.6 \%$ increased risk of having MS than urban residents. With respect to MS components, the crude prevalence of raised fasting glucose and raised blood pressure was significantly greater in rural than in urban participants. However, no significant difference in the prevalence of MS was observed between semi-urban and urban participants.

Conclusions: Rural residents in Shaanxi province, northwest China, were at increased risk of MS, which could be partly explained by sociodemographic and lifestyle differences. In addition, the gap between urban and semi-urban areas seemed to be minimized in related to MS prevalence. Much more attention should be paid to and intervention strategies were needed to address the rural-urban disparities in China.
\end{abstract}

Keywords: Metabolic syndrome, Prevalence, Rural, Urban, China, Cross-sectional survey

\section{Background}

Metabolic syndrome (MS) is defined as a cluster of metabolic abnormalities including abdominal obesity, hyperglycemia, hypertension, and dyslipidemia [1,2]. The patients with MS are at an increased risk of diabetes, cardiovascular disease and subsequent mortality [2-4]. With the increasing obesity and sedentary lifestyle during the past decade [5-7], the prevalence of MS has been rising worldwide [8-10]. Although it's well documented that the MS is

\footnotetext{
* Correspondence: qiuheji@hotmail.com

${ }^{1}$ Department of Endocrinology, Xijing Hospital, Fourth Military Medical University, 169 Changle Road West, Xi'an 710032, China

Full list of author information is available at the end of the article
}

highly prevalent in urban residents, some epidemiological studies suggest that the rural residents are associated with higher or comparative prevalence of MS among adults in the Unites States [11], Korea [12,13] and Thai [14].

Different from the developed provinces in which urbanization has been almost achieved, Shaanxi province, as an undeveloped representative of China, is at an accelerated process of urbanization nowadays, which results in rural industrialization, conversion of farmland, and in situ "urbanized rural residents" [15,16]. The ongoing urbanization offers rural population opportunities not only for economic improvement but also for substantial health risks (air pollution, water hazard, change of diet 
pattern and inadequacy of activities, etc.) [15,16]. Therefore, given that the prevalence of overweight and obesity approximately doubled in rural residents over a 10-year period (1992-2002) and rural adults had a much faster annual increase than rural counterparts (2.52 vs. 0.24) [17], we hypothesized that Chinese rural adults in some places, e.g. undeveloped provinces, may experience an increased risk of MS compared with urban adults. Albeit some researches related to rural-urban difference of MS [18-22], there lacks studies focusing on this point in undeveloped provinces in China.

We utilized the data of the 2007-08 China National Diabetes and Metabolic Disorders Study, aiming to evaluate the rural-urban differences in prevalence of MS and its individual components in Shaanxi province, northwestern China.

\section{Methods}

\section{Study population}

This cross-sectional survey, as part of China National Diabetes and Metabolic disorders Study [23], was conducted from June 2007 to May 2008. A multistage, stratified sampling method was used to select a representative sample of persons with age 20 years or older in the general population in Shaanxi province. In the first stage, a provincial capital (Xi'an), a midsize city (Yan'an), a developed country and an undeveloped country were selected. In the second stage, two city regions from the two cities, plus two country-seats and two rural townships from the two countries were randomly selected. In the third stage, two street districts or rural villages were randomly selected from each urban city district (or country-seat) and rural township, respectively. In the final stage, individuals were randomly sampled stratified by gender and by age distribution based on the 2006 population data of Shaanxi province [24].

The study was primarily designed to provide accurate estimates of the prevalence of diabetes. A sample size of 3,200 individuals was planned to provide an $80 \%$ power to detect a $3 \%$ prevalence of diabetes [with 95\% confidence intervals $(95 \% \mathrm{CI}), \alpha=0.05, \beta=0.2]$, which was sufficient to examine MS due to its higher prevalence compared with diabetes. A total of 3,930 individuals who had lived in their residence for 5 years or longer were randomly chosen and invited. A total of 3,297 individuals completed the survey and examination (the overall response rate: $83.9 \%$ ). After the exclusion of 101 individuals for whom any data of waist circumference, fasting blood glucose, blood pressure, serum triglycerides, and serum high-density lipoprotein cholesterol (HDL-c) were missing, 3,196 individuals were finally included as study participants, of which 1,467 individuals were from urban areas (street district from urban city), 839 from semiurban areas (street district of country-seat), and 890 from rural areas (villages from rural township). The study was approved by the Ethics Committee of Xijing Hospital, Fourth Military Medical University. All the participants signed written informed consent prior to data collection.

\section{Data collection}

A standardized questionnaire was designed and performed by trained nurses or physicians at local health stations or in community clinics to collect data on demographic characteristics, lifestyle risk factors, personal medical history, and family history of diseases [25]. Educational level was categorized as elementary school or uneducated, secondary school, and college or above. Yearly family income was classified as below 10,000 China Yuan (CNY) [1,400 US Dollar (USD)], 10,000-30,000 CNY (1,400-4,200 USD) and above 30,000 CNY (4,200 USD). Cigarette smoking was defined as a lifetime history of smoking at least 100 cigarettes. Alcohol drinking was defined as consuming alcohol at least once per week. Physical activity was defined as participating in moderate or vigorous activity for 30 minutes or more per day at least 3 days a week. Family history of disease was defined as at least one of parents, brothers or sisters diagnosed a disease in a lifetime by selfreporting. Body weight and height were measured without shoes and in light clothing, and body mass index (BMI) calculated as weight in kilograms divided by the square of height in meters. Waist circumference was measured at the middle point between the costal margin and iliac crest. Blood pressure was measured using a standardized mercury sphygmomanometer in the sitting position after at least 5 minutes of rest; two consecutive readings of blood pressure were taken on the same arm and the mean of the 2 measures was used for analysis [23].

After at least 10 hours of overnight fasting, all participants were administered an oral glucose tolerance test to measure blood glucose. A standard 75-g glucose solution was given to participants with no history of diabetes, while a steamed bun that contained approximately $80 \mathrm{~g}$ of complex carbohydrates was given to those with a self-reported history of diabetes for safety reasons. Blood samples were collected at 0,30 , and 120 minutes after the glucose or carbohydrate load to measure blood glucose. Fasting blood samples were also collected to measure serum triglycerides and HDL-c level with the use of an enzymatic method. All laboratory measurements met a standardization and certification program [23].

\section{Definition}

The MS was defined according to the 2009 Joint Interim Statement (2009 JIS) proposed by the International Diabetes Federation Task Force on Epidemiology and Prevention, the National Heart, Lung, and Blood Institute, the American Heart Association, the World Heart Federation, the International Atherosclerosis Society, 
and the International Association for the Study of Obesity, using the Asian criteria for central obesity when $\geq 3$ of the following components were present [2]: 1) larger waist circumference: $\geq 90 \mathrm{~cm}$ (males) or $\geq 80 \mathrm{~cm}$ (females); 2) raised fasting triglycerides: $\geq 1.69 \mathrm{mmol} / \mathrm{l}$ or the use of lipid medications; 3) raised blood pressure: systolic blood pressure $\geq 130 \mathrm{mmHg}$, or diastolic blood pressure $\geq 85 \mathrm{mmHg}$, or the use of antihypertensive medications; 4) raised fasting glucose: $\geq 5.6 \mathrm{mmol} / \mathrm{l}$ or the use of diabetes medications; 5) lowered HDL-c: < $1.04 \mathrm{mmol} / \mathrm{l}$ (male) or $<1.29 \mathrm{mmol} / \mathrm{l}$ (female).

\section{Statistical analysis}

Data analysis was conducted by SPSS 18.0 or Stata 11.0 and data were expressed as mean \pm standard deviation (SD), median with interquartile range, or percentage as suitable. Comparisons between groups were analyzed by $t$-test for measurement data, and chi-square test for enumeration data. $P<0.05$ was defined as the threshold for statistical significance.

Age-standardized prevalence estimates and 95\% confidence intervals (95\% CI) of MS for urban, semi-urban and rural residents stratified by sex were calculated with Stata svy command (direct standardization) to account for the overall population. The calculations were weighted based on the 2006 population distribution of Shaanxi province [24].

Stepwise multivariate analysis was utilized to examine the association between residents' location and MS. The reference group was urban residence. In model 0 , the unadjusted odds ratio (OR) and 95\% CI for MS were calculated. Model 1 was adjusted for age, sex and ethnics; Model 2 was further adjusted for sociodemographic and lifestyle variables including educational level, yearly family income, cigarette smoking, alcohol drinking and physical activities; and model 3 was added for previous factors plus family history of diabetes and hypertension.

\section{Results}

The clinical characteristics of study participants were showed in Table 1 . Rural residents were more likely to have lower education and lower level of yearly family income compared with urban residents. The proportion of alcohol drinking, soft drinks and physical activities were lower in rural residents than in urban residents. Only 9.7\% of rural residents had a family history of diabetes, as compared with a percentage of $13 . \%$ in urban residents. However, there were no significant differences between semi-urban and urban residents except for ethnics, educational level, physical activities, diastolic blood pressure and serum HDL-c level.

The age-standardized prevalence of MS in all, male and female urban participants was $25.9 \%, 27.6 \%$ and $24.4 \%$, respectively. In all and female subgroups, rural participants
Table 1 Clinical characteristics of study participants in Shaanxi province, northwestern China

\begin{tabular}{|c|c|c|c|}
\hline Variable & Urban & Semi-urban & Rural \\
\hline Number & 1467 & 839 & 890 \\
\hline Age, years & $43.8 \pm 14.3$ & $42.7 \pm 13.0$ & $44.3 \pm 13.5$ \\
\hline Sex (male), n (\%) & $625(42.6)$ & 335 (39.9) & $375(42.1)$ \\
\hline Ethnics (Han), n (\%) & $1423(98.7)$ & $821(99.6)^{*}$ & $870(98.2)$ \\
\hline \multicolumn{4}{|l|}{ Educational level, n (\%) } \\
\hline $\begin{array}{l}\text { Elementary school } \\
\text { or below }\end{array}$ & $359(24.9)$ & $142(17.8)^{*}$ & $180(20.3)$ \\
\hline Secondary school & $613(42.6)$ & $335(42.0)$ & $602(67.9)^{* *}$ \\
\hline College or above & $467(32.5)$ & $321(40.2)^{*}$ & $104(11.7)^{* *}$ \\
\hline Yearly family income, n (\%) & $(n=1266)$ & $(n=756)$ & $(n=801)$ \\
\hline 10000 CNY or below & $490(38.7)$ & $307(40.6)$ & $501(62.5)^{* *}$ \\
\hline $10000-30000$ CNY & $571(45.1)$ & $339(44.8)$ & $257(32.1)^{* *}$ \\
\hline 30000 CNY or above & $205(16.2)$ & $110(14.6)$ & $43(5.4)^{* *}$ \\
\hline Cigarette smoking, n (\%) & $348(23.7)$ & $187(22.3)$ & $218(24.5)$ \\
\hline Alcohol drinking, n (\%) & $393(27.0)$ & $210(25.1)$ & $166(18.9)^{* *}$ \\
\hline Physical activities, n (\%) & $568(38.9)$ & $375(44.9)^{*}$ & $257(29.1)^{* *}$ \\
\hline \multicolumn{4}{|l|}{ Soft drinks, n (\%) } \\
\hline Once or below per week & 1165 (81.7) & $699(85.3)$ & $772(88.0)^{* *}$ \\
\hline 2-4 times per week & $159(11.2)$ & $72(8.8)$ & $83(9.5)$ \\
\hline 5 times or above per week & $102(7.2)$ & $48(5.9)$ & $22(2.5)^{* *}$ \\
\hline Family history of DM, n (\%) & $182(13.2)$ & $99(12.6)$ & $68(9.7)^{* *}$ \\
\hline Family history of HT, n (\%) & $528(36.0)$ & $319(38.0)$ & $340(38.2)$ \\
\hline Waist circumference, $\mathrm{cm}$ & $81.8 \pm 10.2$ & $81.5 \pm 9.9$ & $82.7 \pm 10.6^{* *}$ \\
\hline Systolic blood pressure, $\mathrm{mmHg}$ & $119.6 \pm 18.8$ & $118.8 \pm 19.2$ & $125.1 \pm 22.1^{* *}$ \\
\hline Diastolic blood pressure, mmHg & $77.2 \pm 11.3$ & $76.2 \pm 11.1^{*}$ & $76.3 \pm 12.1^{* *}$ \\
\hline Fasting glucose, mmol/l & $5.22 \pm 1.38$ & $5.16 \pm 1.34$ & $5.58 \pm 1.45^{* *}$ \\
\hline Serum triglycerides, mmol/l & $1.58 \pm 1.18$ & $1.53 \pm 0.96$ & $1.49 \pm 1.03$ \\
\hline Serum HDL-c, mmol// & $1.30 \pm 0.30$ & $1.26 \pm 0.30^{*}$ & $1.32 \pm 0.31$ \\
\hline
\end{tabular}

${ }^{*}$ semi-urban vs. urban, $P<0.05$; ${ }^{*}$ rural vs. urban, $P<0.05$; CNY: China Yuan; DM: diabetes mellitus; $\mathrm{HT}$ : hypertension; HDL-c: high-density lipoprotein cholesterol.

had a significant higher prevalence compared with urban counterparts $(29.0 \%$ vs. $25 . \%$ and $30.2 \%$ vs. $24.4 \%$, respectively). However, no significant differences in the prevalence of MS were observed between semi-urban and urban participants in all, male and female subgroups (Table 2).

Comparison between rural and urban participants showed that the prevalence of raised fasting glucose and raised blood pressure was significantly greater in rural than in urban participants among all, male and female subgroups. In females, the prevalence of larger waist circumference was significant higher in rural areas. However, comparison between semi-urban and urban participants showed no significant differences in prevalence of all the individual components among all, male and female subgroups (Table 3). 
Table 2 Crude and age-standardized prevalence of metabolic syndrome among the study participants stratified by sex in Shaanxi province, northwestern China

\begin{tabular}{|c|c|c|c|c|c|c|c|c|}
\hline \multirow{2}{*}{$\begin{array}{l}\text { Age group } \\
\text { year }\end{array}$} & \multicolumn{2}{|c|}{ Urban } & \multicolumn{3}{|c|}{ Semi-urban } & \multicolumn{3}{|c|}{ Rural } \\
\hline & $n / N$ & $\% \pm S D$ & $n / N$ & $\% \pm S D$ & $P$ value & $n / N$ & $\% \pm$ SD & $P$ value \\
\hline \multicolumn{9}{|l|}{ All } \\
\hline $20-30$ & $21 / 267$ & $7.9 \pm 3.3$ & $14 / 143$ & $9.8 \pm 4.9$ & & $7 / 148$ & $4.7 \pm 3.5$ & \\
\hline $30-40$ & $68 / 374$ & $18.2 \pm 3.9$ & $43 / 228$ & $18.9 \pm 5.2$ & & $47 / 205$ & $22.9 \pm 5.8$ & \\
\hline $40-50$ & $101 / 343$ & $29.4 \pm 4.8$ & $55 / 214$ & $25.7 \pm 5.9$ & & $66 / 199$ & $33.2 \pm 6.6$ & \\
\hline $50-60$ & $93 / 228$ & $40.8 \pm 6.4$ & $82 / 174$ & $47.1 \pm 7.5$ & & $112 / 221$ & $50.7 \pm 6.6$ & \\
\hline$\geq 60$ & $130 / 255$ & $51.0 \pm 6.2$ & $36 / 80$ & $45.0 \pm 11.1$ & & $63 / 117$ & $53.8 \pm 9.2$ & \\
\hline Crude & $413 / 1467$ & $28.2 \pm 2.4$ & 230/839 & $27.4 \pm 3.0$ & & 295/890 & $33.1 \pm 3.1$ & \\
\hline Standardized* & & $25.9 \pm 2.1$ & & $24.2 \pm 2.4$ & 0.693 & & $29.0 \pm 2.7$ & 0.017 \\
\hline \multicolumn{9}{|l|}{ Male } \\
\hline $20-30$ & 18/112 & $16.1 \pm 6.9$ & $8 / 61$ & $13.1 \pm 8.7$ & & $3 / 61$ & $4.9 \pm 5.6$ & \\
\hline $30-40$ & $47 / 172$ & $27.3 \pm 6.7$ & $28 / 86$ & $32.6 \pm 10.1$ & & $23 / 85$ & $27.1 \pm 9.6$ & \\
\hline $40-50$ & $35 / 127$ & $27.6 \pm 7.8$ & $26 / 86$ & $30.2 \pm 9.9$ & & $22 / 83$ & $26.5 \pm 9.7$ & \\
\hline $50-60$ & $35 / 94$ & $37.2 \pm 9.9$ & $22 / 64$ & $34.4 \pm 12.0$ & & $38 / 96$ & $39.6 \pm 10.0$ & \\
\hline$\geq 60$ & $46 / 120$ & $38.3 \pm 8.8$ & $15 / 38$ & $39.5 \pm 6.3$ & & $27 / 50$ & $54.0 \pm 14.3$ & \\
\hline Crude & $181 / 625$ & $29.0 \pm 3.6$ & $99 / 335$ & $29.6 \pm 5.0$ & & $113 / 375$ & $30.1 \pm 4.6$ & \\
\hline Standardized* & & $27.6 \pm 3.5$ & & $26.9 \pm 3.7$ & 0.643 & & $27.2 \pm 4.2$ & 0.784 \\
\hline \multicolumn{9}{|l|}{ Female } \\
\hline $20-30$ & $3 / 155$ & $1.9 \pm 2.2$ & $6 / 82$ & $7.3 \pm 5.8$ & & $4 / 87$ & $4.6 \pm 4.5$ & \\
\hline $30-40$ & $21 / 202$ & $10.4 \pm 4.2$ & $15 / 142$ & $10.6 \pm 5.1$ & & $24 / 120$ & $20.0 \pm 7.3$ & \\
\hline $40-50$ & $66 / 216$ & $30.6 \pm 6.1$ & $29 / 128$ & $22.7 \pm 7.3$ & & $44 / 116$ & $37.9 \pm 9.0$ & \\
\hline $50-60$ & $58 / 134$ & $43.3 \pm 8.5$ & $60 / 110$ & $54.5 \pm 9.5$ & & $74 / 125$ & $59.2 \pm 8.7$ & \\
\hline$\geq 60$ & $84 / 135$ & $62.2 \pm 8.3$ & $21 / 42$ & $50.0 \pm 15.8$ & & $36 / 67$ & $53.7 \pm 12.3$ & \\
\hline Crude & $232 / 842$ & $27.6 \pm 3.0$ & $131 / 504$ & $26.0 \pm 3.8$ & & $182 / 515$ & $35.3 \pm 4.1$ & \\
\hline Standardized* & & $24.4 \pm 2.4$ & & $23.6 \pm 3.0$ & 0.842 & & $30.2 \pm 3.5$ & 0.003 \\
\hline
\end{tabular}

*The calculations were weighted based on the 2006 population distribution of Shaanxi province. SD: standard deviation.

The multiple logistic regression models were showed in Table 4. In unadjusted analysis (Model 0), rural participants had 1.237 times higher odds of having MS than urban counterparts (95\% CI: 1.001-1.530). After adjusted for age, sex, and ethnics (Model 1), rural participants still had a significantly higher odds ratio (OR: 1.288, 95\% CI: 1.028-1.613). In the next two steps, after further adjustment for educational level, yearly family income, cigarette smoking, alcohol drinking, and physical activities (Model 2) and family history of diabetes and hypertension (Model 3), respectively, the significance still existed and rural participants finally had a $27.6 \%$ increased risk of having MS than urban adults. Besides, the odds ratios of MS for semi-urban participants compared with urban counterparts showed no statistical significance in all the models.

\section{Discussion}

In this paper, we classified study participants according to their locations as urban, semi-urban and rural residents instead of traditional dichotomy in China. The results showed that the prevalence of MS was significant higher in rural residents than in urban counterparts, in particular among females. After adjusted for the listed risk factors, rural residents had a $27.6 \%$ increased risk of having MS than urban residents. With respect to MS components, the prevalence of raised fasting glucose and raised blood pressure was significantly greater in rural than in urban participants. However, no significant difference in the prevalence of MS was observed between semi-urban and urban participants.

Unlike our findings, previous studies had reported a higher prevalence of MS in urban than in rural areas in China. For instance, Gu D. et al. performed a survey in a nationwide sample of 15,540 Chinese adults aged 3574 years in 2000-01 and indicated that the age-standardized prevalence of MS was higher in urban (18.6\%) than in rural (12.7\%) residents [22]. Lao XQ et al. analyzed a provincial representative sample of 6468 residents aged 20 years or above in Guangdong province during 2002 and the results showed that the urban population had a higher prevalence 
Table 3 Crude prevalence of individual component of metabolic syndrome among the study participants stratified by sex in Shaanxi province, northwestern China

\begin{tabular}{|c|c|c|c|c|c|c|c|c|}
\hline \multirow[t]{2}{*}{ Variable } & \multicolumn{2}{|c|}{ Urban } & \multicolumn{3}{|c|}{ Semi-urban } & \multicolumn{3}{|c|}{ Rural } \\
\hline & $n / N$ & $\% \pm S D$ & $n / N$ & $\% \pm$ SD & $P$ value & $n / N$ & $\% \pm$ SD & $P$ value \\
\hline \multicolumn{9}{|l|}{ All } \\
\hline Raised fasting glucose & $348 / 1467$ & $23.7 \pm 2.2$ & $176 / 839$ & $21.0 \pm 2.7$ & 0.130 & $355 / 890$ & $39.9 \pm 3.2$ & $<0.001$ \\
\hline Raised blood pressure & $504 / 1467$ & $34.4 \pm 2.4$ & $264 / 839$ & $31.5 \pm 3.1$ & 0.157 & $386 / 890$ & $43.4 \pm 3.2$ & $<0.001$ \\
\hline Raised serum triglycerides & $475 / 1467$ & $32.4 \pm 2.4$ & $276 / 839$ & $32.9 \pm 3.2$ & 0.799 & $272 / 890$ & $30.6 \pm 3.0$ & 0.358 \\
\hline Lowered serum HDL-c & $529 / 1467$ & $36.1 \pm 2.4$ & $337 / 839$ & $40.2 \pm 3.2$ & 0.050 & $304 / 890$ & $34.2 \pm 3.1$ & 0.349 \\
\hline Larger waist circumference & $603 / 1467$ & $41.1 \pm 2.5$ & $344 / 839$ & $41.0 \pm 3.3$ & 0.961 & $397 / 890$ & $44.6 \pm 3.3$ & 0.095 \\
\hline \multicolumn{9}{|l|}{ Male } \\
\hline Raised fasting glucose & $139 / 625$ & $22.2 \pm 3.3$ & $84 / 335$ & $25.1 \pm 4.6$ & 0.322 & $148 / 375$ & $39.5 \pm 5.0$ & $<0.001$ \\
\hline Raised blood pressure & $243 / 625$ & $38.9 \pm 3.9$ & $124 / 335$ & $37.0 \pm 5.2$ & 0.571 & $184 / 375$ & $49.1 \pm 5.1$ & $<0.001$ \\
\hline Raised serum triglycerides & $247 / 625$ & $39.5 \pm 3.8$ & $146 / 335$ & $43.6 \pm 5.3$ & 0.223 & $125 / 375$ & $33.3 \pm 4.8$ & 0.050 \\
\hline Lowered serum HDL-c & $170 / 625$ & $27.2 \pm 3.5$ & $100 / 335$ & $29.9 \pm 5.0$ & 0.384 & $97 / 375$ & $25.9 \pm 4.5$ & 0.645 \\
\hline Larger waist circumference & $241 / 625$ & $38.6 \pm 3.9$ & $130 / 335$ & $38.8 \pm 5.2$ & 0.941 & $133 / 375$ & $35.5 \pm 4.8$ & 0.328 \\
\hline \multicolumn{9}{|l|}{ Female } \\
\hline Raised fasting glucose & $209 / 842$ & $24.8 \pm 2.9$ & $92 / 504$ & $18.3 \pm 3.3$ & 0.005 & $207 / 515$ & $40.2 \pm 4.4$ & $<0.001$ \\
\hline Raised blood pressure & $261 / 842$ & $31.0 \pm 3.1$ & $140 / 504$ & $27.8 \pm 3.9$ & 0.211 & $202 / 515$ & $39.2 \pm 4.2$ & 0.002 \\
\hline Raised serum triglycerides & $228 / 842$ & $27.1 \pm 3.0$ & $130 / 504$ & $25.8 \pm 3.8$ & 0.267 & $147 / 515$ & $28.5 \pm 3.9$ & 0.343 \\
\hline Lowered serum HDL-C & $359 / 842$ & $42.6 \pm 3.3$ & $237 / 504$ & $47.0 \pm 4.4$ & 0.117 & $207 / 515$ & $40.2 \pm 4.2$ & 0.376 \\
\hline Larger waist circumference & $362 / 842$ & $43.0 \pm 3.3$ & $214 / 504$ & $42.5 \pm 3.3$ & 0.848 & $264 / 515$ & $51.3 \pm 4.3$ & 0.003 \\
\hline
\end{tabular}

SD: standard deviation; HDL-c: high-density lipoprotein cholesterol.

of MS than the rural population (8.99\% vs. 5.27\%) [19]. Similar finding was also suggested in Weng X.'s [21] and Zhao J.'s [20] surveys which conducted in 2002 and 2006 in eastern China, respectively. However, we found that rural residents had a $27.6 \%$ increased risk of having MS than urban counterparts after adjustment, and raised fasting glucose and raised blood pressure were the two individual components related to the rural-urban difference of MS.

This discrepancy could be partly explained by the fact that our survey was conducted in an undeveloped province within five years while most of previous studies analyzed data from surveys in developed provinces or ten years ago. Shaanxi province, different from developed provinces in which the urbanization has been almost achieved, is at an accelerated process of urbanization. The ongoing urbanization brings rural residents both higher income and an unhealthy lifestyle, as poor early life conditions increase the risk of obesity in a subsequently more socio-economically developed environment [26]. With the expansion of city areas, rural villages are subsumed, large areas of farmlands are converted to urban use, their rural residents are compensated for several sets of housing, and some residents often turn to renting property as an alternative source of income, all of which result in the deficit of working activities in farmlands. Even residents in remote rural villages can also receive financial support from family members in urban areas, which could affect a rural family's agricultural activities. Importantly, this deficit in energy expenditure is unlikely to be compensated for

Table 4 Odds ratios for metabolic syndrome among rural and semi-urban as compared to urban participants in Shaanxi province, northwestern China

\begin{tabular}{|c|c|c|c|c|c|c|c|c|c|}
\hline \multirow[t]{2}{*}{ Variable } & \multirow{2}{*}{$\begin{array}{l}\text { Urban } \\
\text { Ref. }\end{array}$} & \multicolumn{4}{|c|}{ Semi-urban } & \multicolumn{4}{|c|}{ Rural } \\
\hline & & $x^{2}$ & OR & $95 \% \mathrm{Cl}$ & $P$ value & $x^{2}$ & OR & $95 \% \mathrm{Cl}$ & $P$ value \\
\hline Model 0 & 1.000 & 0.002 & 0.995 & $0.806-1.228$ & 0.961 & 3.862 & 1.237 & $1.001-1.530$ & 0.049 \\
\hline Model 1 & 1.000 & 0.066 & 1.029 & $0.824-1.285$ & 0.798 & 4.859 & 1.288 & $1.028-1.613$ & 0.028 \\
\hline Model 2 & 1.000 & 0.111 & 1.038 & $0.831-1.297$ & 0.739 & 4.779 & 1.286 & $1.026-1.610$ & 0.029 \\
\hline Model 3 & 1.000 & 0.088 & 1.034 & $0.829-1.293$ & 0.767 & 4.419 & 1.276 & $1.017-1.601$ & 0.036 \\
\hline
\end{tabular}

OR: odds ratio; $\mathrm{Cl}$ : confidence interval. The covariables in each step enter the model by a forward method. Model 0: unadjusted; Model 1: adjusted for age, sex and ethnic; Model 2: further adjusted for educational level, yearly family income, cigarette smoking, alcohol drinking and physical activities; Model 3: further adjusted for family history of diabetes and family history of hypertension. 
through leisure physical activities [27]. Not to mention that little awareness and knowledge of the risks of inactivity among rural residents worsen inadequacy of leisure physical activities [28]. In addition, rapid urbanization impacts on diet shifts among rural residents, including large increases in energy density, in the proportion of the population consuming a high fat diet and in animal product intake [29]. The direct consequence of inadequacy activity and diet change is the increased prevalence of obesity and metabolic abnormalities (e.g. MS, raised fasting glucose, raised blood pressure), as shown in our study. To some extent, the urbanized rural villages have shared the environmental risk factors of cities (e.g. chemical contamination of foodstuffs, water and air), but not enjoyed the advantage (e.g. available recreational resources, adequate medical insurance, and convenient access to health care services). That's the reason rural-urban difference of MS prevalence in our study persisted even after the adjustment of demographic and lifestyle risk factors, indicating that the listed factors only partly explain and some other factors, such as environmental hazards, may contribute to the regional difference. Actually, previous epidemiological studies also indicated that rural residents may face an increased risk of MS [11-14]. For example, Trivedi T. et al. analyzed the data from the 1999-2006 Nation Health and Nutrition Examination Survey and estimated the prevalence of MS was higher in rural than in urban residents $39.9 \%$ vs. $32.8 \%)$ [11].

Different from the report by Ntandou G [30], no significant difference in the prevalence of MS was observed between semi-urban and urban areas in our study, suggesting that the gap between urban and semi-urban areas seems to be closed due to urbanization in Shaanxi province. Our findings revealed that the two areas face a similar background such as MS prevalence and lifestyle risk factors. In Shaanxi provinces, some educational institutions move to semi-urban areas, which leads to a higher educational level in semi-urban than in urban areas.

Although our study was a multistage, stratified randomly sampling survey, some limitations should be considered. Firstly, the cross-sectional natural decides a possible deficiency of causal inferences. Secondly, urban and women participants were oversampled, which may result in a potential selection bias contributed to the rural-urban difference. Thirdly, risk factors such as dietary intake (or global dietary index), environmental hazards, and diabetes or hypertension knowledge were not assessed for some reasons. We were thus unable to further explore the possible cause of rural-urban difference. Lastly, the data of yearly family income in our survey could not totally reflect the real disposable income which is hard to obtain due to privacy in China.

\section{Conclusions}

The results of the study indicated that rural residents in Shaanxi province, northwest China, were at increased risk of MS, which could be partly explained by sociodemographic and lifestyle differences. Furthermore, the gap between urban and semi-urban areas seemed to be minimized in related to MS prevalence. It's in question whether our study was an isolated issue or would happen in another province. However, it's most likely that China's urbanization will continue unabated. Considering the high prevalence of MS among rural populations and the likelihood of developing medical complications, much more attention should be paid and intervention strategies were needed to address the rural-urban disparities in China.

\section{Competing interests}

The authors declare that they have no conflict of interests.

\section{Authors' contributions}

SX, JM and CY contributed equally to the study. SX, JM had full access to all the data in the study, and took responsibility for the integrity of the data and the accuracy of the analysis. QJ, SX, JM, and CY conceived the study. All authors contributed to the analysis, which was mainly done by SX and JM. SX and CY wrote the first draft, and all authors contributed to the writing of the final report. All authors read and approved the final manuscript.

\section{Acknowledgement}

This study was supported by the Chinese Medical Association Foundation and Chinese Diabetes Society. QJ was supported by the Natural Science Foundation of Shaanxi Province, China (Grant No. 2009ZDKG-76). We thank all physicians and participants of the study, for their co-operations and generous participations.

\section{Author details}

'Department of Endocrinology, Xijing Hospital, Fourth Military Medical University, 169 Changle Road West, Xi'an 710032, China. ${ }^{2}$ Department of Oncology, Xiangyang Central Hospital, Affiliated Hospital of Hubei University of Arts and Science, Xiangyang 441021, China. ${ }^{3}$ Department of Health Statistics, School of Preventive Medicine, Fourth Military Medical University, Xi'an 710032, China. ${ }^{4}$ Institute of Molecular \& Experimental Medicine, School of Medicine, Cardiff University, Health Park, Cardiff CF14 4XN, United Kingdom.

Received: 5 November 2013 Accepted: 31 January 2014 Published: 1 February 2014

\section{References}

1. Alberti KG, Zimmet P, Shaw J: Metabolic syndrome-a new world-wide definition. A consensus statement from the international diabetes federation. Diabet Med 2006, 23(5):469-480.

2. Alberti KG, Eckel RH, Grundy SM, Zimmet PZ, Cleeman Jl, Donato KA, Fruchart JC, James WP, Loria CM, Smith SC Jr, et al: Harmonizing the metabolic syndrome: a joint interim statement of the International Diabetes Federation Task Force on Epidemiology and Prevention; National Heart, Lung, and Blood Institute; American Heart Association; World Heart Federation; International Atherosclerosis Society; and International Association for the study of obesity. Circulation 2009, 120(16):1640-1645.

3. Wu SH, Liu Z, Ho SC: Metabolic syndrome and all-cause mortality: a meta-analysis of prospective cohort studies. Eur J Epidemio/ 2010, 25(6):375-384.

4. Mottillo S, Filion KB, Genest J, Joseph L, Pilote L, Poirier P, Rinfret S, Schiffrin EL, Eisenberg MJ: The metabolic syndrome and cardiovascular risk a systematic review and meta-analysis. J Am Coll Cardiol 2010, 56(14):1113-1132.

5. Stevens GA, Singh GM, Lu Y, Danaei G, Lin JK, Finucane MM, Bahalim AN, McIntire RK, Gutierrez HR, Cowan M, et al: National, regional, and global 
trends in adult overweight and obesity prevalences. Popul Health Metr 2012, 10(1):22

6. Malik VS, Willett WC, Hu FB: Global obesity: trends, risk factors and policy implications. Nat Rev Endocrinol 2013, 9(1):13-27.

7. Knuth AG, Hallal PC: Temporal trends in physical activity: a systematic review. J Phys Act Health 2009, 6(5):548-559.

8. Okafor $\mathrm{Cl}$ : The metabolic syndrome in Africa: current trends. Indian Endocrinol Metab 2012, 16(1):56-66.

9. Mozumdar A, Liguori G: Persistent increase of prevalence of metabolic syndrome among U.S. adults: NHANES III to NHANES 1999-2006. Diabetes Care 2011, 34(1):216-219.

10. Shen J, Goyal A, Sperling L: The emerging epidemic of obesity, diabetes, and the metabolic syndrome in china. Cardiol Res Pract 2012, 2012:178675.

11. Trivedi T, Liu J, Probst JC, Martin AB: The metabolic syndrome: are rural residents at increased risk? J Rural Health 2013, 29(2):188-197.

12. Lim S, Jang HC, Lee HK, Kimm KC, Park C, Cho NH: A rural-urban comparison of the characteristics of the metabolic syndrome by gender in Korea: the Korean Health and Genome study (KHGS). J Endocrinol Invest 2006, 29(4):313-319.

13. Kim TN, Kim JM, Won JC, Park MS, Lee SK, Yoon SH, Kim HR, Ko KS, Rhee BD: A decision tree-based approach for identifying urban-rural differences in metabolic syndrome risk factors in the adult Korean population. J Endocrinol Invest 2012, 35(9):847-852.

14. Aekplakorn W, Kessomboon P, Sangthong R, Chariyalertsak S, Putwatana P, Inthawong $R$, Nitiyanant $W$, Taneepanichskul $S$ : Urban and rural variation in clustering of metabolic syndrome components in the Thai population: results from the fourth National Health Examination Survey 2009. BMC Public Health 2011, 11:854.

15. Gong P, Liang S, Carlton EJ, Jiang Q, Wu J, Wang L, Remais JV: Urbanisation and health in China. Lancet 2012, 379(9818):843-852.

16. Zhu YG, loannidis JP, Li H, Jones KC, Martin FL: Understanding and harnessing the health effects of rapid urbanization in China. Environ SCi Technol 2011, 45(12):5099-5104

17. Wang Y, Mi J, Shan XY, Wang QJ, Ge KY: Is China facing an obesity epidemic and the consequences? The trends in obesity and chronic disease in China. Int J Obes (Lond) 2007, 31(1):177-188.

18. Xi B, He D, Hu Y, Zhou D: Prevalence of metabolic syndrome and its influencing factors among the Chinese adults: The China Health and Nutrition Survey in 2009. Prev Med 2013, 57(6):867-871.

19. Lao XQ, Zhang YH, Wong MC, Xu YJ, Xu HF, Nie SP, Ma WJ, Thomas GN, Yu IT: The prevalence of metabolic syndrome and cardiovascular risk factors in adults in southern China. BMC Public Health 2012, 12:64.

20. Zhao J, Pang ZC, Zhang L, Gao WG, Wang SJ, Ning F, Nan HR, Ren J, Qiao Q: Prevalence of metabolic syndrome in rural and urban Chinese population in Qingdao. J Endocrinol Invest 2011, 34(6):444-448.

21. Weng X, Liu Y, Ma J, Wang W, Yang G, Caballero B: An urban-rural comparison of the prevalence of the metabolic syndrome in Eastern China. Public Health Nutr 2007, 10(2):131-136.

22. Gu D, Reynolds K, Wu X, Chen J, Duan X, Reynolds RF, Whelton PK, He J: Prevalence of the metabolic syndrome and overweight among adults in China. Lancet 2005, 365(9468):1398-1405.

23. Yang W, Lu J, Weng J, Jia W, Ji L, Xiao J, Shan Z, Liu J, Tian H, Ji Q, et al: Prevalence of diabetes among men and women in China. N Engl J Med 2010, 362(12):1090-1101.

24. National Bureau of Statistics of China: China statistical yearbook - 2006. Beijing: China Statistics Press; 2006 [Accessed February 26, 2010, at http:// www.stats.gov.cn/tjsj/ndsj/2006/indexeh.htm.]

25. Luepker RV, Evans A, McKeigue P, Reddy KS: Cardiovascular survey methods. 3rd edition. Geneva: World Health Organization; 2004

26. Kavikondala S, Schooling CM, Jiang CQ, Zhang WS, Cheng KK, Lam TH, Leung GM: Pathways to obesity in a developing population: the Guangzhou biobank cohort study. Int J Epidemiol 2009, 38(1):72-82.

27. Bauman A, Allman-Farinelli M, Huxley R, James WP: Leisure-time physical activity alone may not be a sufficient public health approach to prevent obesity-a focus on China. Obes Rev 2008, 9(Suppl 1):119-126.

28. Haase A, Steptoe A, Sallis JF, Wardle J: Leisure-time physical activity in university students from 23 countries: associations with health beliefs, risk awareness, and national economic development. Prev Med 2004, 39(1):182-190
29. Popkin BM, Du S: Dynamics of the nutrition transition toward the animal foods sector in China and its implications: a worried perspective. J Nutr 2003, 133(11 Suppl 2):3898S-3906S.

30. Ntandou G, Delisle H, Agueh V, Fayomi B: Abdominal obesity explains the positive rural-urban gradient in the prevalence of the metabolic syndrome in Benin. West Africa. Nutr Res 2009, 29(3):180-189.

doi:10.1186/1471-2458-14-104

Cite this article as: Xu et al.: Urban, semi-urban and rural difference in the prevalence of metabolic syndrome in Shaanxi province, northwestern China: a population-based survey. BMC Public Health 2014 14:104.

\section{Submit your next manuscript to BioMed Central and take full advantage of:}

- Convenient online submission

- Thorough peer review

- No space constraints or color figure charges

- Immediate publication on acceptance

- Inclusion in PubMed, CAS, Scopus and Google Scholar

- Research which is freely available for redistribution 